\title{
Integration over complex manifolds via Hochschild homology
}

\author{
Ajay C. Ramadoss
}

\begin{abstract}
Given a holomorphic vector bundle $\mathcal{E}$ on a connected compact complex manifold $X$, in [FLS] a $\mathbb{C}$-linear functional $I_{\mathscr{E}}$ on $\mathrm{H}^{2 n}(X, \mathbb{C})$ is constructed. This is done by producing a linear functional on the 0 -th completed Hochschild homology $\widehat{\mathrm{HH}}_{0}(\mathscr{D i f f}(\mathcal{E}))$ of the sheaf of holomorphic differential operators on $\mathcal{E}$ using topological quantum mechanics. It is shown in [FLS] that this functional is $\int_{X}$ if $\mathscr{E}$ has non-zero Euler characteristic, and the conjecture is that it is $\int_{X}$ for all $\mathcal{E}$.

In a subsequent work [Ram] the author proved that the linear functional $I_{\mathcal{E}}$ is independent of the vector bundle $\mathcal{E}$. This article builds upon the work in [Ram] to prove that $I_{\mathcal{E}}=\int_{X}$ for an arbitrary holomorphic vector bundle $\mathcal{E}$ on an arbitrary connected compact complex manifold $X$. This is done using an argument that is very natural from the geometric point of view. Moreover, this argument enables one to make the approach to this conjecture developed first in [FLS] and subsequently in [Ram] independent of the Riemann-Roch-Hirzebruch theorem. This argument allows us to extend the construction in [FLS] to a construction of a linear functional $I_{\mathcal{E}}$ on $\mathrm{H}_{c}^{2 n}(Y, \mathbb{C})$ for a holomorphic vector bundle $\mathcal{E}$ with bounded geometry on an arbitrary connected complex manifold $Y$ with bounded geometry, and to prove that $I_{\mathcal{E}}=\int_{Y}$. We also generalize a result of $[\mathrm{Ram}]$ pertaining to "cyclic homology analogs" of $I_{\mathcal{E}}$.
\end{abstract}

Mathematics Subject Classification (2000). 16E40.

Keywords. Completed Hochschild homology, heat kernel, trace class operator, supertrace, differential operators, soft sheaves.

\section{Introduction}

Let $X$ be a smooth, connected compact complex manifold, and let $\&$ be a holomorphic vector bundle on $X$. In what follows, the term vector bundle shall refer to a holomorphic vector bundle over a complex manifold unless explicitly stated otherwise. Let $\operatorname{Diff}(\mathcal{E})$ be the sheaf of holomorphic differential operators on $\mathcal{E}$. We have a notion of completed Hochschild homology $\widehat{\mathrm{HH}}$. $(\mathscr{D i f f}(\mathcal{E}))$ such that there is an isomorphism

$$
\beta_{\mathcal{E}}: \widehat{\mathrm{HH}}_{-i}(\operatorname{Diff}(\mathcal{E})) \simeq \mathrm{H}^{2 n-i}(X, \mathbb{C})
$$

for every integer $i$. In [FLS] B. Feigin, A. Losev and B. Shoikhet describe the construction of a $\mathbb{C}$-linear functional tr on $\widehat{\mathrm{HH}}_{0}(\mathcal{D i f f}(\mathcal{E}))$ using topological quantum 
mechanics. Denote the linear functional $\operatorname{tr} \circ \beta_{\mathcal{E}}^{-1}$ on $\mathrm{H}^{2 n}(X, \mathbb{C})$ by $I_{\mathcal{E}}$. We call $I_{\mathcal{E}}$ the FLS functional of $\mathcal{E}$. We also have a notion of completed cyclic homology $\widehat{\mathrm{HC}}$. $(\mathscr{D i f f}(\mathcal{E}))$ such that

$$
\widehat{\mathrm{HC}}_{-j}(\operatorname{Diff}(\mathcal{E})) \simeq \mathrm{H}^{2 n-j}(X, \mathbb{C}) \oplus \mathrm{H}^{2 n-j+2}(X, \mathbb{C}) \oplus \cdots .
$$

The construction of $\operatorname{tr}$ can be generalized to provide linear functionals $\operatorname{tr}_{2 i}$ on $\widehat{\mathrm{HC}}_{-2 i}(\mathscr{D i f f}(\mathcal{E}))$ for each $i \geq 0$. The linear functional $\operatorname{tr}_{2 i}$ therefore yields a linear functional $I_{\mathcal{E}, 2 i, 2 k}$ on $\mathrm{H}^{2 n-2 k}(X, \mathbb{C})$ whenever $0 \leq k \leq i$.

In [FLS] it is shown that if $\mathscr{E}$ is a vector bundle of non-zero Euler characteristic, then $I_{\mathcal{E}}=\int_{X}$ as linear functionals on $\mathrm{H}^{2 n}(X, \mathbb{C})$. This is done using the RiemannRoch-Hirzebruch theorem. In [FLS] it is conjectured that $I_{\mathcal{E}}=\int_{X}$ in general. We refer to this conjecture as the integral conjecture for the rest of this article. In [Ram] it is demonstrated that $I_{\mathcal{E}}=I_{\mathcal{F}}$ for any two holomorphic vector bundles $\mathcal{E}$ and $\mathscr{F}$ on $X$. We may therefore refer to $I_{\mathcal{O}_{X}}$ as the FLS functional on $X$. The fact that $I_{\mathcal{E}}$ is independent of $\mathscr{E}$ implies that $I_{\mathcal{E}}=\int_{X}$ as long as $\mathcal{E}$ is a holomorphic vector bundle on a compact complex manifold $X$ that admits at least one vector bundle of non-zero Euler characteristic. This proved the integral conjecture for compact complex manifolds arising from complex algebraic varieties, since smooth complex algebraic variety has at least one vector bundle of non-zero Euler characteristic (see the introduction in [Ram] for an argument proving this assertion). The integral conjecture for arbitrary compact complex manifolds, however, remained an open question as it is not known whether or not there exist compact complex manifolds with no holomorphic vector bundle of non-zero Euler characteristic.

The purpose of this article is to prove the integral conjecture in general. This is done by building upon the work in [Ram] which, in turn, is a further development of the approach to this problem in [FLS]. The argument used here is very natural from the geometric point of view. Furthermore, it makes the approach to the integral conjecture developed in [FLS] and subsequently in [Ram] independent of the Riemann-RochHirzebruch theorem.

Convention. Throughout this paper, a connected complex manifold shall mean a connected complex manifold that is complete and has bounded geometry (positive radius of injectivity plus all covariant derivatives of the Ricci curvature are bounded) as a Riemannian manifold.

Our approach to the integral conjecture in this note also enables us to extend the construction of the FLS functional to the construction of a $\mathbb{C}$-linear functional $I_{\mathcal{E}}$ on $\mathrm{H}_{c}^{2 n}(Y, \mathbb{C})$ given a vector bundle $\mathscr{E}$ with bounded geometry (see Definition on p. 40) on an arbitrary connected complex manifold $Y$, and to prove that $I_{\mathcal{E}}=\int_{Y}$ (Theorem 1). Here $\mathrm{H}_{c}^{*}$ denotes cohomology with compact supports. One can also extend the construction of $I_{\mathcal{E}, 2 i, 2 k}$ for vector bundles on compact complex manifolds to vector bundles on arbitrary complex manifolds. Given a vector bundle $\&$ with bounded geometry on an arbitrary connected complex manifold $Y$, one can extend 
the construction of $I_{\mathcal{E}, 2 i, 2 k}$ from the compact complex case to construct a $\mathbb{C}$-linear functional $I_{\mathcal{E}, 2 i, 2 k}$ on $\mathrm{H}_{c}^{2 n-2 k}(Y, \mathbb{C})$. Strengthening a result of [Ram], we show that $I_{\mathcal{E}, 2 i, 0}=\int_{Y}$ and $I_{\mathcal{E}, 2 i, 2 k}=0$ whenever $k>0$ (Theorem 2).

Outline of this note. Section 1 contains certain remarks about the idea used in this note. This may help the reader understand the motivation behind this article better.

Section 2 recalls the construction of the FLS functional on $X$.

In Section 3 the integral conjecture is proved in general and the construction of the FLS functional is extended to vector bundles with bounded geometry on arbitrary connected complex manifolds.

Acknowledgements. I am very grateful to Prof. Boris Tsygan for going through this paper carefully and for his very useful comments and suggestions. I also thank the referee for the careful reading of this article and for the helpful remarks and advice.

\section{Some remarks}

Remark 1. We note that the argument used to prove the integral conjecture in Section 3.1 shows that to prove the integral conjecture for an arbitrary compact complex manifold of complex dimension $n$, it suffices to prove it for one compact complex manifold of the same complex dimension. This observation enables us to free this approach from the Riemann-Roch-Hirzebruch theorem (see Section 3.2). Recall that the proof of the integral conjecture for vector bundles of non-zero Euler characteristic uses the fact that the class of the global differential operator id in $\mathrm{H}^{2 n}(X, \mathbb{C})$ is $\left(\mathrm{Ch}(\mathcal{E}) \cdot \mathrm{Td}_{X}\right)_{2 n}$ (see [NT1] and [NT2]) along with the Riemann-Roch-Hirzebruch theorem. It turns out that, by Theorem 2 of [Ram] together with the argument in Section 3.1, we need this only for one particular vector bundle on one particular compact complex manifold of dimension $n$ for the integral conjecture to hold for every compact complex manifold of dimension $n$. This is exploited in Section 3.2 to do away with the need for the Riemann-Roch-Hirzebruch theorem altogether. A special case of the fact that the class of the global differential operator id in $\mathrm{H}^{2 n}(X, \mathbb{C})$ is $\left(\mathrm{Ch}(\mathcal{E}) \cdot \mathrm{Td}_{X}\right)_{2 n}$ is, however, still used. This fact together with the integral conjecture implies the Riemann-Roch-Hirzebruch theorem itself, giving yet another proof of the Riemann-Roch-Hirzebruch theorem.

Remark 2. The argument in Section 3.1 is also very natural from the geometric point of view. Let $U$ be an open disc with inclusions into two compact complex manifolds $X$ and $Y$. If $\omega$ is a top degree differential form on $U$ supported compactly in $U$, then

$$
\int_{X} \omega=\int_{U} \omega=\int_{Y} \omega
$$


The crux of this article is to prove "directly" that the Feigin-Losev-Shoikhet linear functional mimics the above behavior of the integral. This is exploited along with the results from [Ram] to prove the integral conjecture in general. We also note that the same idea is behind the extension of the Feigin-Losev-Shoikhet construction of the integral via topological quantum mechanics to non-compact complex manifolds as well (Section 3.3). Of course, cohomology with compact supports has to be used instead of cohomology itself.

Remark 3. A related conjecture in [FLS] that has since been proven in [EnFe] pertained to traces of global holomorphic differential operators on $\mathcal{E}$. If $D$ is a global holomorphic differential operator on $\mathcal{E}, D$ induces endomorphisms on $\mathrm{H}^{i}(X, \mathcal{E})$ for all $i$. The supertrace of $D, \operatorname{str}(D)$, is given by the formula

$$
\operatorname{str}(D)=\sum_{i}(-1)^{i} \operatorname{tr}\left(\left.D\right|_{\mathrm{H}^{i}(X, \mathcal{E})}\right) .
$$

Further, $D$ is seen to yield a class $[D]$ in $\widehat{\mathrm{HH}}_{0}(\mathscr{D i f f}(\mathcal{E}))$ (see [FLS], [Ram]). It follows from the construction of tr: $\widehat{\mathrm{HH}}_{0}(\mathscr{D i f f}(\mathcal{E})) \rightarrow \mathbb{C}$ (see [FLS], [Ram]) that

$$
\operatorname{tr}([D])=\operatorname{str}(D) .
$$

Denote the element $\beta_{\mathcal{E}}([D])$ of $\mathrm{H}^{2 n}(X, \mathbb{C})$ by $[D]$ itself. It was conjectured in [FLS] and proven in $[\mathrm{EnFe}]$ that

$$
\operatorname{str}([D])=\int_{X}[D]
$$

We shall refer to this result as the supertrace theorem. It is somewhat similar to Corollary 5.6 of [S-S]. Note that the integral conjecture implies the supertrace theorem. Also note that the supertrace theorem together with Theorem 2 of [Ram] implies the integral conjecture for any compact complex manifold that admits at least one holomorphic vector bundle admitting at least one global holomorphic differential operator with non-zero supertrace. Unfortunately, we do not know whether every compact complex manifold has this property. We also point out that by proving the integral conjecture in full generality, this article completes a different "RiemannRoch-Hirzebruch theorem free" approach to the supertrace theorem from that in [EnFe]. Proposition 4.1 of [EnFe] inspired us to use a "heat kernel" approach to push the idea outlined in Remark 2 through.

\section{Preliminary material}

This section is meant to briefly recall the salient aspects of earlier work in [FLS] and $[\mathrm{Ram}]$. For further details, the reader is referred to [FLS] and [Ram]. Let 
$\operatorname{Dolb}\left(X, \mathcal{O}_{X}\right)$ denote the Dolbeaux resolution of $\mathcal{O}_{X}$. Denote by $\operatorname{Diff}^{\bullet}(\mathcal{E})$ the complex $\operatorname{Diff}(\mathscr{E}) \otimes \mathcal{O}_{X} \operatorname{Dolb}\left(X, \mathcal{O}_{X}\right)$. Let Diff ${ }^{\bullet}(\mathcal{E})$ denote the differential graded algebra of global sections of $\operatorname{Diff}^{\bullet}(\mathcal{E})$. Let $K_{\mathscr{E}}^{\bullet}$ denote the complex $\Gamma\left(X, \mathcal{E} \otimes \mathcal{O}_{X} \operatorname{Dolb}\left(X, \mathcal{O}_{X}\right)\right)$. By basic Hodge theory (see [Vois], Theorem 5.24), $K_{\mathscr{E}}^{\bullet}$ splits into a direct sum of a complex $K_{0 \mathscr{E}}^{\bullet}$ of $\mathbb{C}$-vector spaces with 0 differential and an acyclic complex $K_{1 \mathcal{E}}$.

2.1. The key construction of [FLS]. The reader may consult [FLS] for further details regarding any assertion made in this section. The key construction of [FLS] is of an $A_{\infty}$-morphism $\mathcal{F}$ from $\operatorname{Diff}{ }^{\bullet}(\mathcal{E})$ to $\operatorname{End}\left(K_{0 \mathcal{E}}\right)$. The $A_{\infty}$-morphism $\mathcal{F}$ induces a map $\widetilde{F}_{\text {Hoch }}$ from the Hochschild chain complex of $\operatorname{Diff}{ }^{\bullet}(\mathcal{E})$ to that of $\operatorname{End}\left(K_{0 \mathcal{E}}^{\bullet}\right)$. One thus obtains a map $\widetilde{F}_{H_{\text {Hoch }}}$ from the Hochschild homology of Diff ${ }^{\bullet}(\mathcal{E})$ to that of $\operatorname{End}\left(K_{0 \varepsilon}^{\bullet}\right)$. Let $\mathrm{HH}_{i}(A)$ denote the $i$-th Hochschild homology of a graded algebra $A$. Then

$$
\begin{aligned}
& \operatorname{HH}_{i}\left(\operatorname{End}\left(K_{0 \varepsilon}^{\bullet}\right)\right) \simeq 0 \text { for all } i \neq 0 \\
& \mathrm{HH}_{0}\left(\operatorname{End}\left(K_{0 \varepsilon}^{\bullet}\right)\right) \simeq \mathbb{C} .
\end{aligned}
$$

The only Hochschild 0 -cycles that have nontrivial images in $\mathrm{HH}_{0}\left(\operatorname{End}\left(K_{0 \varepsilon}^{\bullet}\right)\right)$ are those arising out of degree 0 elements of $\operatorname{End}\left(K_{0 \mathcal{E}}^{\bullet}\right)$. The image in $\mathbb{C}$ of the class in $\mathrm{HH}_{0}\left(\operatorname{End}\left(K_{0 \varepsilon}^{\bullet}\right)\right)$ of a Hochschild 0 cycle arising out of a degree 0 element $M$ of $\operatorname{End}\left(K_{0 \varepsilon}^{\bullet}\right)$ is the supertrace $\operatorname{str}(M)$ of $M$. We therefore denote the identification of $\mathrm{HH}_{0}\left(\operatorname{End}\left(K_{0 \mathcal{E}}^{\bullet}\right)\right)$ with $\mathbb{C}$ by str. It follows from this and from the formula for $\widetilde{F}_{\text {Hoch }}$ (see [FLS]) that if $a$ is a degree $k-1$ element of $\operatorname{Diff}^{\bullet}(\mathcal{E})^{\otimes k}$ yielding a Hochschild 0 -cycle of $\operatorname{Diff}^{\bullet}(\mathcal{E})$, then

$$
\operatorname{tr}\left(\mathcal{F}_{\text {Hoch }^{*}}(a)\right)=\sum_{j=0}^{j=k-1} \operatorname{str}\left(\mathcal{F}_{k}\left(\tau^{j}(a)\right)\right) .
$$

In the above equation, $\tau$ is the $\mathbb{C}$-endomorphism of $\operatorname{Diff}^{\bullet}(\mathcal{E})^{\otimes k}$ arising out of a cyclic permutation of factors with the appropriate sign, and $\widetilde{F}_{k}$ is the $k$-th Taylor component of the $A_{\infty}$-morphism $\mathcal{F}$ (for more details, see [FLS]).

We now describe the construction of the Taylor components $\mathcal{F}_{k}$ of $\mathcal{F}$.

2.1.1. The Taylor components of $\mathscr{F}$. Suppose that $C_{k}$ is the configuration space $\left\{t_{1}<\cdots<t_{k} \mid t_{i} \in \mathbb{R}\right\} / G^{(1)}$ where $G^{(1)}$ is the one-dimensional group of shifts $\left(t_{1}, \ldots, t_{k}\right) \rightarrow\left(t_{1}+c, \ldots, t_{k}+c\right)$. This is a smooth $(k-1)$-dimensional manifold which is not compact if $k>1$. Note that setting $\tau_{i}:=t_{i+1}-t_{i}$ identifies the $C_{k}$ with the open orthant $\prod_{i=1}^{i=k-1}\left\{\tau_{i}>0\right\}$. Let $\overline{\left\{\tau_{i}>0\right\}}$ denote the compactification of $\left\{\tau_{i} \geq 0\right\}$ by a point at infinity. Let $\overline{C_{k}}=\prod_{i=1}^{i=k-1} \overline{\left\{\tau_{i}>0\right\}}$. This is a compactification of $C_{k}$. 
If $\phi$ is an element of $\operatorname{End}\left(K_{\mathscr{E}}^{\bullet}\right)$, let $[\phi]_{i}$ denote the endomorphism id $\otimes \cdots \otimes \phi \otimes$ $\cdots \otimes$ id of $\left(K_{\mathcal{E}}^{*}\right)^{\otimes k}$ where $\phi$ acts on the $i$-th factor from the right. Recall that $\bar{\partial}_{\mathcal{E}}^{*}$ denotes the Hodge adjoint of $\bar{\partial}_{\mathcal{E}}$. Similarly, $\Delta_{\mathcal{E}}$ denotes the Laplacian of $\bar{\partial}_{\mathcal{E}}$.

Let $\Phi$ denote the differential form

$$
[\mathrm{id}]_{k} \circ\left[\exp \left[-d \tau_{k-1} \bar{\partial}_{\mathcal{E}}^{*}-\tau_{k-1} \Delta_{\mathcal{E}}\right]\right]_{k-1} \circ \cdots \circ\left[\exp \left[-d \tau_{1} \bar{\partial}_{\mathcal{E}}^{*}-\tau_{1} \Delta_{\mathcal{E}}\right]\right]_{1}
$$

on $C_{k}$ with values in $\operatorname{End}\left(K_{\mathcal{E}}^{\bullet}\right)^{\otimes k}\left(\right.$ note that $\left.\operatorname{End}\left(\left(K_{\mathcal{E}}^{\bullet}\right)^{\otimes k}\right) \simeq \operatorname{End}\left(K_{\mathcal{E}}^{\bullet}\right)^{\otimes k}\right)$. This extends to a differential form on $\overline{C_{k}}$ with values in $\operatorname{End}\left(K_{\mathcal{E}}^{\bullet}\right)^{\otimes k}$. In addition, there is a composition map from $\operatorname{End}\left(K_{\mathcal{E}}^{\bullet}\right)^{\otimes k}$ to $\operatorname{End}\left(K_{\mathcal{E}}^{\bullet}\right)$ which we shall denote by $\mathrm{m}_{k}$. An element $D$ of $\operatorname{Diff}^{\bullet}(\mathscr{E})^{\otimes k}$ yields an element of $\operatorname{End}\left(K_{\mathscr{E}}^{\bullet}\right)^{\otimes k}$ which shall also be denoted by $D$. If $\mathcal{I}$ and $\Pi$ denote the inclusion of $K_{0 \mathcal{E}}^{\bullet}$ as a direct summand of $K_{\mathcal{E}}^{\bullet}$ and the projection from $K_{\mathcal{E}}^{\bullet}$ to $K_{0 \mathcal{E}}^{\bullet}$ respectively, then

$$
\mathscr{F}_{k}(D)=\int_{\overline{C_{k}}} \Pi \circ \mathrm{m}_{k}(\Phi \circ D) \circ \mathcal{I}=\Pi \circ\left(\int_{\overline{C_{k}}} \mathrm{~m}_{k}(\Phi \circ D)\right) \circ \mathcal{I} .
$$

That the $\mathscr{F}_{k}$ form the Taylor components of an $A_{\infty}$-morphism is shown in [FLS].

2.2. A linear functional on $\widehat{\mathbf{H H}}_{\mathbf{0}}(\mathscr{D i f f}(\mathcal{E}))$. For an open subset $U$ of $X$, let $\operatorname{Diff}(\mathcal{E})(U)$ and $\operatorname{Diff}^{\bullet}(\mathcal{E})(U)$ denote by $\Gamma(U, \operatorname{Diff}(\mathcal{E}))$ and $\Gamma\left(U, \operatorname{Diff}^{\bullet}(\mathcal{E})\right)$, respectively. Let $C^{\bullet}(\operatorname{Diff}(\mathscr{E})(U))$ denote the complex of Hochschild chains of $\operatorname{Diff}(\mathscr{E})(U)$ (converted into a cochain complex). We note that the Hochschild differential on $C^{\bullet}(\operatorname{Diff}(\mathscr{E})(U))$ extends to a differential of degree 1 on the graded vector space $\bigoplus_{k \geq 1} \operatorname{Diff}\left(\varepsilon^{\bowtie k}\right)\left(U^{k}\right)[k-1]$ where $\mathcal{E}^{\otimes k}$ is the $k$-fold external tensor power of $\mathcal{E}$ on $X^{k}$. We denote the resulting complex by $\left.\overline{C^{\bullet}(\operatorname{Diff}(\mathcal{E})(U)}\right)$. Similarly, we note that the Hochschild differential on $C^{\bullet}\left(\right.$ Diff $\left.^{\bullet}(\mathcal{E})(U)\right)$ extends to a differential of degree 1 on the graded vector space $\bigoplus_{k \geq 1} \operatorname{Diff}^{\bullet}\left(\mathcal{E}^{\otimes k}\right)\left(U^{k}\right)[k-1]$. We denote the resulting complex by $\overline{C^{\bullet}\left(\operatorname{Diff}^{\bullet}(\mathcal{E})(U)\right)}$. Let $\overline{C^{\bullet}(\mathscr{D i f f}(\mathcal{E}))}$ denote the sheaf of complexes associated to the pre-sheaf

$$
U \leadsto \overline{C^{\bullet}(\operatorname{Diff}(\mathcal{E})(U))}
$$

of complexes of $\mathbb{C}$-vector spaces on $X$. Similarly, let $\widehat{\operatorname{Hoch}(\mathscr{D i f f}(\mathscr{E}))}$ denote the sheaf of complexes associated to the pre-sheaf

$$
U \leadsto \overline{C^{\bullet}\left(\operatorname{Diff}^{\bullet}(\mathcal{E})(U)\right)}
$$

of complexes of $\mathbb{C}$-vector spaces on $X$.

By definition, $\left.\widehat{\mathrm{HH}}_{i}(\operatorname{Diff}(\mathcal{E}))=\mathbb{M}^{i}\left(X, \overline{C^{\bullet}(\operatorname{Diff}(\mathcal{E})}\right)\right)$.

Alas, $\mathcal{F}$ does not automatically yield a map of complexes from $\overline{\operatorname{Hoch}(\mathscr{D i f f}(\mathcal{E}))}$ to $C^{\bullet}\left(\operatorname{End}\left(K_{0 \mathcal{E}}^{\bullet}\right)\right)$. One has, however, the following facts (see Proposition 6 of [Ram]). 
Recall that any 0-cocycle $\alpha$ of $C^{\bullet}\left(\operatorname{Diff}^{\bullet}(\mathcal{E})\right)$ is of the form $\sum_{k} \alpha_{k}$ where $\alpha_{k} \in$ $\operatorname{Diff}^{\bullet}(\mathscr{E})^{\otimes k}[k-1]$. Note that $\alpha_{1} \in \operatorname{Diff}^{0}(\mathcal{E})$ and $\alpha_{k}=0$ for almost all $k$. Let $\Pi_{0}$ denote the projection from $K_{\mathcal{E}}^{\bullet}$ onto the kernel of the Laplacian $\Delta_{\mathcal{E}}: K_{\mathscr{E}}^{\bullet} \rightarrow K_{\mathscr{E}}^{\bullet}$. This is an integral operator with smooth kernel (see [BGV] Chapter 2).

Fact 1. The linear functionals

$$
\alpha \mapsto \sum_{k} \sum_{j=0}^{j=k-1} \operatorname{str}\left(\widetilde{F}_{k}\left(\tau^{j}\left(\alpha_{k}\right)\right)\right) \quad \text { and } \quad \alpha \mapsto \operatorname{str}\left(\Pi_{0} \alpha_{1} \Pi_{0}\right)
$$

coincide on the space of 0 -cocycles of $C^{\bullet}\left(\operatorname{Diff}^{\bullet}(\mathcal{E})\right)$. Denote this linear functional by $I_{\mathrm{FLS}}$. Recall from [FLS] that $I_{\mathrm{FLS}}$ vanishes on 0 -coboundaries.

Fact 2 . The linear functional $I_{\mathrm{FLS}}$ extends to a linear functional on the 0 -th cohomology of $\Gamma(X, \widehat{\operatorname{Hoch}(\operatorname{Diff}(\mathcal{E}))})$. We will denote it by tr.

On the other hand, the natural degree preserving map of complexes from $\left.\overline{C^{\bullet}(\mathscr{D i f f}(\mathscr{E})}\right)$ to $\widehat{\operatorname{Hoch}(\mathscr{D i f f}(\mathcal{E}))}$ is a quasi-isomorphism since Diff ${ }^{\bullet}\left(\mathcal{E}^{\otimes k}\right)\left(U^{k}\right)$ is quasi-isomorphic to $\operatorname{Diff}\left(\varepsilon^{\otimes k}\right)\left(U^{k}\right)$ for any $k \geq 1$ and any open subset $U$ of $X$. Also, $\widehat{\operatorname{Hoch}(\mathscr{D i f f}(\mathcal{E}))}$ is a complex of sheaves of $\mathbb{C}$-vector spaces that are modules over the sheaf of smooth functions on $X$. It follows that the $i$-th cohomology of the complex $\Gamma(X, \widehat{\operatorname{Hoch}(\mathscr{D i f f}(\mathscr{E})}))$ is $\left.\left.\mathbb{\boxplus}^{i}(X, \widehat{\operatorname{Hoch}(\mathscr{D i f f}(\mathcal{E})})\right)=\mathbb{\boxplus}^{i}\left(X, \widehat{C^{\bullet}(\mathscr{D i f f}(\mathscr{E})}\right)\right)=$ $\widehat{\mathrm{HH}}_{i}(\operatorname{Diff}(\mathcal{E}))$.

It follows that tr is a linear functional on $\widehat{\mathrm{HH}}_{0}(\mathscr{D i f f}(\mathcal{E}))$. Also recall (for instance, [Ram] Lemma 3) that $\overline{C^{\bullet}(\mathscr{D i f f}(\mathscr{E}))}$ is quasi-isomorphic to the shifted constant sheaf $\mathbb{C}[2 n]$. It follows that $\widehat{\mathrm{HH}}_{-i}(\mathscr{D i f f}(\mathcal{E})) \simeq \mathrm{H}^{2 n-i}(X, \mathbb{C})$. Moreover, $\widehat{\text { tr }}$ yields a linear functional on $\mathrm{H}^{2 n}(X, \mathbb{C})$, which we denote by $I_{\mathcal{E}}$.

\section{Generalizing the integral conjecture}

Let $X$ be a compact complex manifold admitting at least one holomorphic vector bundle of non-zero Euler characteristic. Let $\mathcal{E}$ be a homomorphic vector bundle on $X$. Let $I_{\mathcal{E}}: \mathrm{H}^{2 n}(X, \mathbb{C}) \rightarrow \mathbb{C}$ be as in the introduction. Since we have already shown in $[\mathrm{Ram}]$ that $I_{\mathcal{E}}=I_{\mathcal{F}}$ for any vector bundle $\mathcal{F}$ on $X$, we may assume without loss of generality that $\mathscr{E}=\mathcal{O}_{X}$. Let $K_{X}^{\bullet}$ denote the Dolbeaux complex of $\mathcal{O}_{X}$. Let $C_{k}$ and $\overline{C_{k}}$ be as in Section 2.1.1.

Let $\operatorname{Diff}(X)$ denote $\operatorname{Diff}\left(\mathcal{O}_{X}\right)$. Choose open discs $U, W$ with $U \subset W \subset X$. Since $\overline{\operatorname{Hoch}(\mathscr{D i f f}(X))}$ is a complex of soft sheaves quasi-isomorphic to $\mathbb{C}[2 n]$, the complex $\Gamma_{c}(U, \widehat{\operatorname{Hoch}(\mathscr{D i f f}(X))})$ is quasi-isomorphic to $\mathrm{H}_{c}^{2 n+\bullet}(U, \mathbb{C})$. Here $\Gamma_{c}$ is the functor "sections with compact support" and $\mathrm{H}_{c}^{*}$ denotes cohomology with compact support. Note that $\mathrm{H}_{c}^{2 n}(U, \mathbb{C}) \simeq \mathbb{C}$ and $\mathrm{H}_{c}^{i}(U, \mathbb{C})=0$ for all $i \neq 2 n$. 
It follows that

$$
\left.\mathrm{H}^{0}\left(\Gamma_{c}(U, \widehat{\operatorname{Hoch}(\mathscr{D i f f}(X)})\right)\right) \simeq \mathrm{H}_{c}^{2 n}(U, \mathbb{C}) \simeq \mathbb{C} .
$$

For any 0 -cocycle $\alpha$ of $\Gamma_{c}(U, \widehat{\operatorname{Hoch}(\mathscr{D i f f}(X))})$, let $[\alpha]_{U}$ denote the class of $\alpha$ in $\mathrm{H}_{c}^{2 n}(U, \mathbb{C})$. We state the following obvious fact as a proposition for emphasis.

Proposition 1. There is a Hochschild 0 -cycle $\alpha$ of $\Gamma_{c}(U, \widehat{\operatorname{Hoch}(\mathscr{D i f f}(X))})$ such that $[\alpha]_{U} \neq 0$ in $\left.\mathrm{H}^{0}\left(\Gamma_{c}(U, \widehat{\operatorname{Hoch}(\mathscr{D i f f}(X)})\right)\right) \simeq \mathrm{H}_{c}^{2 n}(U, \mathbb{C})$.

Let $\alpha$ be as in Proposition 1. Let $\Delta$ denote the Laplacian of $\bar{\partial}$ on $K_{X}^{\bullet}$. Let $K_{X L^{2}}^{\bullet}$ denote the Hilbert space of square integrable Dolbeaux forms on $X$. This is a $\mathbb{Z}_{2}$-graded Hilbert space. Let $D^{k}$ denote sheaf associated to the pre-sheaf $U \leadsto$ $\operatorname{Diff}^{\bullet}\left(U^{k}\right)[k-1]$. Let $\alpha_{k}$ denote the component of $\alpha$ in $\Gamma_{c}\left(U, D^{k}\right)$. Note that $\alpha_{k}=0$ for almost all $k$, and that $\alpha_{1}$ is a compactly supported element of $\operatorname{diff}^{0}(U)$. Therefore, $\alpha_{1}$ may also be thought of as an element of $\operatorname{diff}^{0}(X)$.

We now recall Proposition 2.45 of [BGV] as a lemma.

Lemma 1. For any scalar $t>0$, the operator $\alpha_{1} \mathrm{e}^{-t \Delta}$ makes sense as a trace class operator on $K_{X L^{2}}^{\bullet}$.

Thus, if

$$
\varphi(\alpha):=\alpha_{1},
$$

then $\varphi(\alpha) \mathrm{e}^{-t \Delta}$ makes sense as a trace class operator on $K_{X L^{2}}^{\bullet}$. Let $\operatorname{str}_{X}(\theta)$ denote the supertrace of $\theta$ for any trace class operator $\theta$ on $K_{X L^{2}}^{\cdot}$. Note that $\alpha$ may also be thought of as a 0 -cocycle of $\Gamma(X, \widehat{\operatorname{Hoch}(\mathscr{D i f f}(X))})$. Let $[\alpha]_{X}$ denote the class of $\alpha$ in $\mathrm{H}^{2 n}(X, \mathbb{C})$. If $j_{X}$ denotes the inclusion from $U$ into $X$, then $[\alpha]_{X}=j_{X *}[\alpha]_{U}$.

\section{Proposition 2.}

$$
\lim _{t \rightarrow \infty} \operatorname{str}_{X}\left(\varphi(\alpha) \mathrm{e}^{-t \Delta}\right)=\int_{X}[\alpha]_{X}
$$

Proof. Let $K_{0 X}^{\bullet}$ denote the kernel of $\Delta$. Recall that $K_{0 X}^{\bullet}$ is finite dimensional. Recall that $\Delta$ is an operator on $K_{X L^{2}}^{*}$ with discrete non-negative spectrum that preserves the $\mathbb{Z}_{2}$-grading. One can thus find a graded Hilbert space basis of $K_{X L^{2}}^{\bullet}$ made up entirely of eigenvectors of $\Delta$. Let $\left\{e_{1}, \ldots, e_{n}, \ldots\right\}$ be such a basis with $\lambda_{i}$ denoting the eigenvalue of $e_{i}$. Let $\langle$,$\rangle denote the inner product of K_{X L^{2}}^{\cdot}$. We will denote $\varphi(\alpha)$ by $\varphi$ for the remainder of this proof.

Then

$$
\operatorname{str}_{X}\left(\varphi \mathrm{e}^{-t \Delta}\right)=\sum_{i} \pm\left\langle\varphi \mathrm{e}^{-t \Delta}\left(e_{i}\right), e_{i}\right\rangle=\sum_{i} \pm \mathrm{e}^{-(t-1) \lambda_{i}}\left\langle\varphi \mathrm{e}^{-\Delta}\left(e_{i}\right), e_{i}\right\rangle
$$


for any $t>1$. Note that the above sums converge absolutely by the fact that $\varphi \mathrm{e}^{-t \Delta}$ is a trace class operator for any $t>0$. It follows that

$$
\begin{aligned}
\lim _{t \rightarrow \infty} \operatorname{str}_{X}\left(\varphi \mathrm{e}^{-t \Delta}\right) & =\lim _{t \rightarrow \infty} \sum_{i} \pm \mathrm{e}^{-(t-1) \lambda_{i}}\left\langle\varphi \mathrm{e}^{-\Delta}\left(e_{i}\right), e_{i}\right\rangle \\
& =\sum_{\left\{i \mid \lambda_{i}=0\right\}} \pm\left\langle\varphi\left(e_{i}\right), e_{i}\right\rangle .
\end{aligned}
$$

The last sum is a finite sum and is equal to $\operatorname{str}\left(\Pi_{K_{0 X}^{\bullet}} \circ \varphi \circ \mathcal{I}_{K_{0 X}^{\cdot}}\right)$ where $\Pi_{K_{0 X}^{\bullet}}$ and $\mathcal{I}_{K_{0 X}^{\bullet}}$ are the projection from $K_{X}^{\bullet}$ to $K_{0 X}^{\bullet}$ and the inclusion from $K_{0 X}^{\bullet}$ into $K_{X}^{\bullet}$, respectively. Since $X$ has at least one vector bundle of non-zero Euler characteristic, it follows that

$$
\operatorname{str}\left(\Pi_{K_{0 X}^{\cdot}} \circ \varphi \circ \mathcal{I}_{K_{0 X}^{\cdot}}\right)=\int_{X}[\alpha]_{X}
$$

by [Ram], Theorem 2. This completes the proof.

Note that

$$
K_{X L^{2}}^{\bullet}=K_{U L^{2}}^{\bullet} \oplus K_{X \backslash U L^{2}}^{\bullet},
$$

as graded Hilbert spaces. Let $\Delta_{U}$ denote the restriction of $\Delta_{X}$ to $U$.

Proposition 3. $\varphi(\alpha) \mathrm{e}^{-t \Delta_{U}}$ is an operator with trace on $K_{U L^{2}}^{\cdot}$ and

$$
\operatorname{str}_{X}\left(\varphi(\alpha) \mathrm{e}^{-t \Delta_{X}}\right)=\operatorname{str}_{U}\left(\varphi(\alpha) \mathrm{e}^{-t \Delta_{U}}\right)
$$

for any $t>0$.

Proof. Denote $\varphi(\alpha)$ by $\varphi$ in this proof. Recall from [BGV] that $\mathrm{e}^{-t \Delta_{X}}$ is an operator with smooth kernel $p_{t}$ (called the heat kernel) and that

$$
\operatorname{str}_{X}\left(\varphi \mathrm{e}^{-t \Delta_{X}}\right)=\int_{X} \operatorname{str}\left(\varphi p_{t}(x, x)\right)|d x|=\int_{U} \operatorname{str}\left(\varphi p_{t}(x, x)\right)|d x|=\operatorname{str}_{U}\left(\varphi \mathrm{e}^{-t \Delta_{U}}\right) .
$$

The last equality holds because the heat kernel on $U$ is unique (see [Don]). The construction of the heat kernel for a non-compact Riemannian manifold is done by modifying the construction in [BGV] for the compact case. As in [Don] this modification goes through, provided that the manifold in question has bounded geometry. The second equality above holds since $\varphi$ is a differential operator supported compactly in $U$.

Recall that $[\alpha]_{U}$ denotes the class of $\alpha$ in $\mathrm{H}_{c}^{2 n}(U, \mathbb{C})$.

\section{Corollary 1.}

$$
\lim _{t \rightarrow \infty} \operatorname{str}_{U}\left(\varphi(\alpha) \mathrm{e}^{-t \Delta}\right)=\int_{U}[\alpha]_{U}
$$


Proof. Since $\alpha$ is compactly supported on a subset of $U$, we have $\int_{X}[\alpha]_{X}=\int_{U}[\alpha]_{U}$. The corollary now follows from Proposition 3 and Proposition 2.

3.1. Proof of the integral conjecture in general. Let $Y$ be an arbitrary compact complex manifold with $\operatorname{dim}_{\mathbb{C}} Y=\operatorname{dim}_{\mathbb{C}} X$. Note that we can find an open disc on $Y$ that we can identify (holomorphically) with $W$. Let $W_{X}$ and $W_{Y}$ denote $W$ thought of as open subdiscs of $X$ and $Y$, respectively. Moreover, the Hermitian metric on $\Omega^{0, \bullet}\left(W_{Y}\right)$ can be chosen such that it coincides with that on $\Omega^{0 \bullet^{\bullet}}\left(U_{X}\right)$ on $U_{Y}$.

Let $j_{X}$ and $j_{Y}$ denote the inclusions from $U$ into $X$ and $Y$, respectively. Let $\alpha$ be as in Proposition 1. Denote $\varphi(\alpha)$ by $\varphi$ in this subsection. Then

$$
\int_{U}[\alpha]_{U}=\int_{X} j_{X_{*}}[\alpha]_{U}=\int_{Y} j_{Y_{*}}[\alpha]_{U}
$$

On the other hand, we have

$$
\operatorname{str}_{X}\left(\varphi \mathrm{e}^{-t \Delta_{X}}\right)=\operatorname{str}_{U}\left(\varphi \mathrm{e}^{-t \Delta_{U}}\right)=\operatorname{str}_{Y}\left(\varphi \mathrm{e}^{-t \Delta_{Y}}\right)
$$

for any $t>0$ by Proposition 3. Taking the limit as $t \rightarrow \infty$ and applying Corollary 1 , we get

$$
\lim _{t \rightarrow \infty} \operatorname{str}_{Y}\left(\varphi \mathrm{e}^{-t \Delta_{Y}}\right)=\lim _{t \rightarrow \infty} \operatorname{str}_{U}\left(\varphi \mathrm{e}^{-t \Delta_{U}}\right)=\int_{U}[\alpha]_{U} .
$$

By (3), we have

$$
\lim _{t \rightarrow \infty} \operatorname{str}_{Y}\left(\varphi \mathrm{e}^{-t \Delta_{Y}}\right)=\int_{Y} j_{Y_{*}}[\alpha]_{U} .
$$

But $[\alpha]_{Y}=j_{Y_{*}}[\alpha]_{U}$, where $\alpha$ on the left-hand side is viewed as a 0 -cocycle of $\Gamma(Y, \widehat{\operatorname{Hoch}(\mathscr{D i f f}(Y))})$. Now, following the proof of Proposition 2, we see that

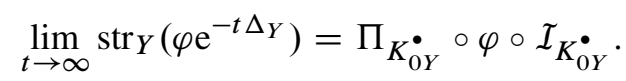

The right-hand side is precisely the Feigin-Losev-Shoikhet linear functional on $Y$ applied to $[\alpha]_{Y}$. It follows that the Feigin-Losev-Shoikhet linear functional on $Y$ applied to $[\alpha]_{Y}$ is precisely $\int_{Y}[\alpha]_{Y}$. Since $[\alpha]_{Y}=j_{Y_{*}}[\alpha]_{U} \neq 0$, this proves that the Feigin-Losev-Shoikhet linear functional on $Y$ is exactly $\int_{Y}$.

3.2. Proving the integral conjecture without the Riemann-Roch-Hirzebruch theorem. Let $Z$ and $Y$ be two compact complex manifolds of complex dimension $n$. The proof in Section 3.1 also shows that the following holds.

Theorem A. The integral conjecture holds for $Z$ iff it holds for $Y$.

Consider the vector bundle $\mathcal{O}_{\mathbb{P} 1}$ on $\mathbb{P}_{\mathbb{C}}^{1}$. Consider the differential operator id on $\mathcal{O}_{\mathbb{P} 1}$. The following special case of the supertrace theorem uses a result from [NT1] and a hands-on calculation. 


\section{Proposition 4.}

$$
1=\operatorname{str}(\mathrm{id})=\int_{\mathbb{P} 1}[\mathrm{id}]
$$

Proof. Let $T_{\mathbb{P} 1}$ denote the tangent bundle of $\mathbb{P}^{1}$. That $\operatorname{str}(\mathrm{id})=1$ follows from the fact that $\mathrm{H}^{0}\left(\mathbb{P}^{1}, \mathcal{O}_{\mathbb{P} 1}\right) \simeq \mathbb{C}$ and $\mathrm{H}^{0}\left(\mathbb{P}^{1}, \mathcal{O}_{\mathbb{P} 1}\right)=0$. By Theorem 7.1.1 of [NT1], [id] $=\left(\operatorname{Td}\left(T_{\mathbb{P} 1}\right)\right)_{2}$. We therefore need to verify that $\int_{\mathbb{P}^{1}}\left(\operatorname{Td}\left(T_{\mathbb{P}^{1}}\right)\right)_{2}=1$. Note that $T_{\mathbb{P} 1}$ is a line bundle. It follows that $\left(\operatorname{Td}\left(T_{\mathbb{P} 1}\right)\right)_{2}=\frac{1}{2} \mathrm{c}_{1}\left(T_{\mathbb{P} 1}\right)$ where $\mathrm{c}_{1}(\mathcal{E})$ denotes the first Chern class of $\mathcal{E}$. Also, $T_{\mathbb{P} 1}=\mathcal{O}(2)$. It therefore suffices to show that $\int_{\mathbb{P} 1} c_{1}(\mathcal{O}(1))=1$. Then $\mathrm{c}_{1}(\mathcal{O}(1))$ is the class of the Chern form of $\mathcal{O}(1)$, which we will denote by $\omega_{\mathrm{Ch}}$. Let $z$ denote the local holomorphic coordinate on an affine line $U \subset \mathbb{P}^{1}$. Then $\int_{\mathbb{P}^{1}} \omega_{\mathrm{Ch}}=\int_{U} \omega_{\mathrm{Ch}}$. On the other hand, on $U$ we have

$$
\omega_{\mathrm{Ch}}=\frac{i}{2 \pi} \frac{d z \wedge d \bar{z}}{\left(1+|z|^{2}\right)^{2}}
$$

by Lemma 3.16 of [Vois]. Setting $z=x+i y$, it follows that

$$
\int_{U} \omega_{\mathrm{Ch}}=\frac{1}{\pi} \int_{\mathbb{R}^{2}} \frac{d x d y}{\left(1+x^{2}+y^{2}\right)^{2}}=1 .
$$

This proves the proposition.

It follows that the operator $\mathrm{id}^{\otimes n}$ on $\mathcal{O}_{\mathbb{P} 1} \times n$ also has supertrace 1. Further, by Proposition 5 below, after identifying $\mathrm{H}^{2 n}\left(\mathbb{P}^{1^{\times n}}, \mathbb{C}\right)$ with $\mathrm{H}^{2}\left(\mathbb{P}^{1}, \mathbb{C}\right)^{\otimes n}$, we obtain that

$$
1=\int_{\mathbb{P}^{\times n}}\left[\mathrm{id}^{\otimes n}\right]=\int_{\mathbb{P} 1^{\times n}}[\mathrm{id}]^{\otimes n} .
$$

The integral conjecture therefore holds for $\mathbb{P}^{1 \times n}$, and hence (by Theorem A) for any compact complex manifold, provided that we prove the following result. In the following proposition, $Y$ and $Z$ are compact complex manifolds. $\mathscr{D}_{1}$ and $\mathscr{D}_{2}$ are global holomorphic operators on $Y$ and $Z$, respectively. As a result $\mathscr{D}_{1} \otimes \mathscr{D}_{2}$ is a global holomorphic differential operator on $Y \times Z$.

\section{Proposition 5.}

$$
\left[\mathscr{D}_{1} \otimes \mathscr{D}_{2}\right]=\left[\mathscr{D}_{1}\right] \otimes\left[\mathscr{D}_{2}\right]
$$

Proof. Step 1: Fixing basic notation.

Let $n$ and $m$ denote the complex dimensions of $Y$ and $Z$ respectively. Let $\overline{C^{\bullet}(\operatorname{Diff}(M))}$ denote the completed Hochschild chain complex $\overline{C^{\bullet}\left(\operatorname{Diff}^{\left(\mathcal{O}_{M}\right)}\right)}$ for any complex manifold $M$ (converted into a cochain complex). We recall from [Bryl] that $\overline{C^{\bullet}(\mathscr{D i f f}(M))}$ is quasi-isomorphic to the shifted constant sheaf $\mathbb{C}[2 d]$ on $M$, where $d$ is the complex dimension of $M$. Denote this quasi-isomorphism by $i_{M}$. 
Let $C^{\bullet}(A)$ denote the Hochschild chain complex of an $\mathbb{C}$-algebra $A$ viewed as a cochain complex. Recall that if $A$ and $B$ are $\mathbb{C}$-algebras, the shuffle product $\amalg$ yields a map of complexes from $C^{\bullet}(A) \otimes C^{\bullet}(B)$ to $C^{\bullet}(A \otimes B)$ (see [Lod], Section 4.2). In particular if $U$ and $V$ are open discs in $Y$ and $Z$ respectively, the shuffle product yields a map of complexes from $C^{\bullet}(\operatorname{Diff}(U)) \otimes C^{\bullet}(\operatorname{Diff}(V))$ to $C^{\bullet}(\operatorname{Diff}(U \times V))$. This further extends to a map of complexes of sheaves of $\mathbb{C}$-vector spaces on $Y \times Z$ from $\overline{C^{\bullet}(\operatorname{Diff}(Y))} \otimes \overline{C^{\bullet}(\operatorname{Diff}(Z))}$ to $\left.\overline{C^{\bullet}(\operatorname{Diff}(Y \times Z)}\right)$, which we will denote by $\mathrm{m}_{\mathrm{Sh}}$.

Step 2: Reduction to a "local check".

Think of $\mathscr{D}_{1}$ and $\mathscr{D}_{2}$ as elements of $\left.\Gamma\left(Y, \overline{C^{0}(\operatorname{Diff}(Y)}\right)\right)$ and $\Gamma\left(Z, \overline{\left.C^{0}\left(D_{\operatorname{iff}(Z)}\right)\right)}\right.$ respectively. Then

$$
\left.\mathrm{m}_{\mathrm{Sh}}\left(\mathscr{D}_{1} \otimes \mathscr{D}_{2}\right)=\mathscr{D}_{1} \otimes \mathscr{D}_{2} \in \Gamma\left(Y \times Z, \overline{C^{0}(\operatorname{Diff}(Y \times Z)}\right)\right) .
$$

To prove this proposition, it therefore suffices to show that the following diagram commutes in the derived category $\mathrm{D}\left(\operatorname{Sh}_{\mathbb{C}}(Y \times Z)\right)$ of sheaves of $\mathbb{C}$-vector spaces on $Y \times Z$ :

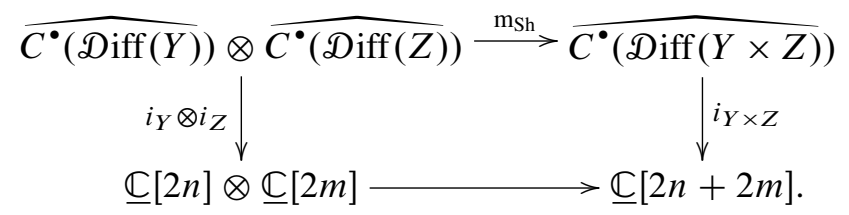

Since a sheaf of $\mathbb{C}$-vector spaces is injective iff it is flabby (see [Riet], Lemma 3.3), the constant sheaf $\mathbb{C}$ is an injective object in the category of $\mathbb{C}$-vector spaces on $X$. It follows from this that the diagram above commutes in $\mathrm{D}\left(\operatorname{Sh}_{\mathbb{C}}(Y \times Z)\right)$ up to a scalar. Checking that that scalar factor is one is "done locally". Let $U$ and $V$ be open discs in $Y$ and $Z$, respectively. It suffices to show that the following diagram commutes up to cohomology in the category of complexes of $\mathbb{C}$-vector spaces:

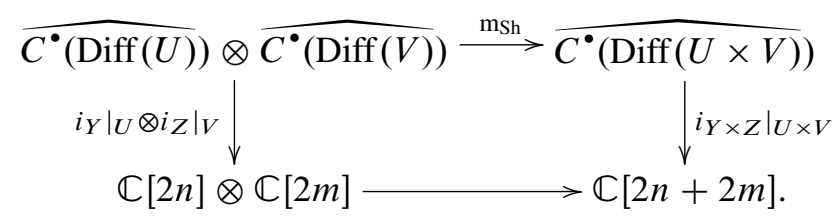

The bottom row of the above diagram is the natural identification of $\mathbb{C} \otimes \mathbb{C}$ with $\mathbb{C}$ that takes $1 \otimes 1$ to 1 .

Step 3: The "local check".

Let $\operatorname{sgn}(\sigma)$ denote the sign of a permutation $\sigma \in S_{k}$. Note that if $W$ is any $\mathbb{C}$-vector space, then $\sigma$ acts on $W^{\otimes k}$ on the right as

$$
\sigma\left(w_{1} \otimes \cdots \otimes w_{k}\right)=w_{\sigma(1)} \otimes \ldots \otimes w_{\sigma(k)} .
$$


Let $\omega(z)$ denote the Hochschild 2-cycle

$$
1 \otimes \frac{\partial}{\partial z} \otimes z-1 \otimes z \otimes \frac{\partial}{\partial z}+1 \otimes 1 \otimes 1
$$

of the Weyl algebra generated by $z$ and $\frac{\partial}{\partial z}$. Then

$$
\omega_{2 n}:=\omega\left(z_{1}\right) \amalg \omega\left(z_{2}\right) \amalg \cdots \amalg \omega\left(z_{n}\right)
$$

is a Hochschild $2 n$-cycle of the Weyl algebra $\mathcal{A}_{n}$ generated by $z_{1}, \ldots, z_{n}$ and $\frac{\partial}{\partial z_{1}}, \ldots, \frac{\partial}{\partial z_{n}}$. If $z_{1}, \ldots, z_{n}$ are local holomorphic coordinates on $U$, then $\mathcal{A}_{n}$ is a subalgebra of $\operatorname{Diff}(U)$. It follows that $\omega_{2 n}$ is a Hochschild $2 n$-cycle in $C^{\bullet}(\operatorname{Diff}(U))$. Note that that image of $\omega_{2 n}$ in the normalized Hochschild chain complex of $\operatorname{Diff}(U)$ is the normalized Hochschild $2 n$-cycle

$$
\sum_{\sigma \in S_{2 n}} \operatorname{sgn}(\sigma) 1 \otimes \sigma\left(\frac{\partial}{\partial z_{1}} \otimes z_{1} \otimes \cdots \otimes \frac{\partial}{\partial z_{n}} \otimes z_{n}\right) .
$$

We recall from $[\mathrm{BrGe}]$ and $[\mathrm{FT}]$ that

$$
\left.i_{Y}\right|_{U}\left(\left[\omega_{2 n}\right]\right)=1 .
$$

To check that the above diagram commutes, we only need to verify that

$$
\mathrm{m}_{\mathrm{Sh}}\left(\omega_{2 n} \otimes \omega_{2 m}\right)=\omega_{2 n+2 m} .
$$

This is immediate from our definition of $\omega_{2 n}$.

Remark. The construction of $\left.i_{Y}\right|_{U}$ from [Bryl] is what we used in [Ram]. Even with this construction if $\left.i_{Y}\right|_{U}$, we can directly verify that $\left.i_{Y}\right|_{U}\left(\left[\omega_{2 n}\right]\right)=1$. We now sketch how this can be done. Recall that the cohomology of $\overline{C^{\bullet}(\operatorname{Diff}(U))}$ is computed using the spectral sequence arising out of the filtration induced by a specific filtration $F^{\circ}$ on $\operatorname{Diff}(U)$. Here $F^{-k} \operatorname{Diff}(U)$ is the space of differential operators on $U$ of order at most $k$. Let $z_{1}, \ldots, z_{n}$ be local holomorphic coordinates on the cotangent bundle $T^{*} U$ of $U$. Let $y_{1}, \ldots, y_{n}$ be local holomorphic coordinates on the fibre of $T^{*} U$. Setting the weight of the $d z_{i}$ to be 0 and that of the $d y_{i}$ to be 1 enables us to define the notion of the weight of a holomorphic form on $T^{*} U$. In the next paragraph, differential forms on $T^{*}(U)$ shall always refer to holomorphic differential forms on $T^{*} U$ that are algebraic along the fibres.

The $E_{1}^{p, q}$-term of the spectral sequence computing the cohomology of $\overline{C^{\bullet}(\operatorname{Diff}(U))}$ is precisely the space of $-p-q$-forms on $T^{*} U$ of weight $-p$ that are algebraic along the fibres. In fact, the image of the cycle $\omega_{2 n}$ in $E_{1}^{-n,-n}$ can be verified to be the differential form $d y_{1} \wedge d z_{1} \wedge d y_{2} \wedge d z_{2} \wedge \ldots \wedge d y_{n} \wedge d z_{n}$. Recall from [Bryl] (Theorem 3.1.1) that the differential on the $E_{1}^{\bullet, \bullet}$-terms is the differential of the canonical 
complex of the Poisson manifold $T^{*} U$. Moreover the canonical complex of $T^{*} U$ may be identified with the (shifted) de Rham complex of $T^{*} U$. Under this identification, $d y_{1} \wedge d z_{1} \wedge d y_{2} \wedge d z_{2} \wedge \cdots \wedge d y_{n} \wedge d z_{n}$ is identified with 1 . This is a de Rham 0 -cocycle. It follows that the image of $\omega_{2 n}$ in $E_{2}^{-n,-n}$ is 1 . Since $E_{2}^{p, q}=0$ whenever $(p, q) \neq(-n,-n)$, the cohomology of $\overline{C^{\bullet}(\operatorname{Diff}(U))}$ is identified with $E_{2}^{-n,-n} \simeq \mathbb{C}$. This shows that $\left.i_{Y}\right|_{U}\left(\left[\omega_{2 n}\right]\right)=1$.

\subsection{The Feigin-Losev-Shoikhet construction for vector bundles on non-compact} complex manifolds. As a byproduct of this proof, we have in fact extended the construction of the Feigin-Losev-Shoikhet linear functionals associated with certain holomorphic bundles on complex manifolds to complex manifolds that are not compact. Let $\mathcal{E}$ be a holomorphic vector bundle on an arbitrary connected complex manifold $Y$. Let $\Delta_{\mathcal{E}}$ denote the Laplacian of $\mathcal{E}$. This depends on a choice of Hermitian metric for $Y$ as well as for $\mathcal{E}$. Recall that the Laplacian $\Delta_{\mathcal{E}}=\Delta^{\mathcal{E}}+F$, where $\Delta^{\mathcal{E}}$ is the Laplacian of a connection on $\mathcal{E}$ (see Definition 2.4 of [BGV]) and $F \in \Gamma(Y, \operatorname{End}(\mathcal{E}))$.

Definition. We say that $\mathcal{E}$ has bounded geometry if for some choice of Hermitian metric on $\mathcal{E}$, there exists a connection $\nabla_{\mathcal{E}}$ on $\mathcal{E}$ such that $\Delta_{\mathcal{E}}=\Delta^{\mathcal{E}}+F$, where $\Delta^{\mathcal{E}}$ is the Laplacian of $\nabla_{\mathcal{E}}$ and $F \in \Gamma(Y, \operatorname{End}(\mathcal{E}))$, and all covariant derivatives of the curvature of $\Delta^{\mathcal{E}}$ as well as of $F$ are bounded on $Y$.

Let $\mathcal{E}$ be a vector bundle having bounded geometry. Let $K_{\mathcal{E}_{L^{2}}}$ denote the $\left(\mathbb{Z}_{2}\right.$-graded) Hilbert space of square integrable sections of $K_{\mathcal{E}}^{\cdot}$. Then $\mathrm{e}^{-t \Delta_{\mathcal{E}}}$ can be constructed as an integral operator on $K_{\mathcal{E} L^{2}}^{\bullet}$ following [Don]. Suppose that $\left.\alpha \in \Gamma_{c}(Y, \widehat{\operatorname{Hoch}(\mathscr{D i f f}(\mathcal{E})})\right)$, and let $D^{k}(\mathcal{E})$ denote the sheaf associated with the presheaf $U \leadsto \operatorname{Diff}^{\bullet}\left(\mathcal{E}^{\square k}\right)\left(U^{k}\right)$. Let $\alpha_{k}$ denote the component of $\alpha$ in $\Gamma_{c}\left(Y, D^{k}(\mathscr{E})\right)$. Note that $\alpha_{k}=0$ for almost all $k$. Put

$$
\varphi(\alpha)=\alpha_{1} .
$$

The following result generalizes Proposition 6 of [Ram].

Proposition 6. (1) $\varphi(\alpha) \mathrm{e}^{-t \Delta_{\mathcal{E}}}$ makes sense as a trace class operator on $K_{\mathcal{E} L^{2}}^{\bullet}$ for any $t>0$.

(2) Furthermore, the map

$$
\alpha \leadsto \lim _{t \rightarrow \infty} \operatorname{str}\left(\varphi(\alpha) \mathrm{e}^{-t \Delta_{\mathcal{E}}}\right)
$$

induces $a \mathbb{C}$-linear functional on $\left.\mathrm{H}^{0}\left(\Gamma_{c}(Y, \overline{\operatorname{Hoch}(\mathscr{D i f f}(\mathscr{E})})\right)\right)$. 
Proof. Step 1: To prove part (1), note that the support of $\alpha$ can be covered by finitely many open discs $U_{1}, \ldots, U_{m} \subset Y$ such that each $U_{i}$ is contained in an open disc $W_{i} \subset Y$ with $\mathcal{E}$ is trivial on each $W_{i}$. Let $Z=Y \backslash \bigcup_{i} \bar{U}_{i}$. One can find a partition of unity $\left\{f_{1}, \ldots, f_{m}, f\right\}$ on $Y$ subordinate to the cover $Y=\cup_{i} U_{i} \cup Z$ with $f_{i}$ supported on $U_{i}$. Note that the support of $f_{i}$ is compact. Also, $f=0$ on the support of $\alpha$. Writing $\alpha$ as $\sum_{i} f_{i} \alpha$ it suffices to prove part (1) for $\alpha$ compactly supported on an open disc $U$ contained in an open disc $W$ on which $\mathcal{E}$ is trivial. One may find a compact complex manifold $X$ containing an open disc $W_{X}$ with which $W$ can be identified holomorphically. Let $\varepsilon^{\prime}=\mathcal{O}_{X}^{p}$, where $p$ is the rank of $\varepsilon$. The metrics on $X$ and $\varepsilon^{\prime}$ may be chosen to coincide with those of $U$ and $\left.\mathcal{E}\right|_{U}$, respectively, on $U_{X}$. Let $\alpha_{X}$ denote $\alpha$ thought of as an element of $\Gamma\left(X, \widehat{\operatorname{Hoch}\left(\mathscr{D i f f}\left(\mathcal{E}^{\prime}\right)\right)}\right)$. By an easy generalization of Lemma $1, \varphi\left(\alpha_{X}\right) \mathrm{e}^{-t \Delta_{\mathcal{E}^{\prime}}}$ makes sense as a trace class operator on $K_{\mathscr{E}^{\prime} L^{2}}^{\bullet}$ for any $t>0$. Since $\alpha_{X}$ is supported on a compact subset of $U_{X}$ and since $K_{\mathcal{E}^{\prime} L^{2}}^{\bullet}=K_{\left.\mathcal{E}^{\prime}\right|_{U_{X}} L^{2}}^{\bullet} \oplus K_{\left.\mathcal{E}^{\prime}\right|_{X \backslash U_{X}} L^{2}}^{\bullet}$, an easy extension of Proposition 3 implies that $\varphi\left(\alpha_{X}\right) \mathrm{e}^{-t \Delta_{\mathcal{E}^{\prime}}}$ makes sense as a trace class operator on $K_{\left.\mathcal{E}^{\prime}\right|_{U_{X}} L^{2}}$ for any $t>0$, and that

$$
\operatorname{str}_{X}\left(\varphi\left(\alpha_{X}\right) \mathrm{e}^{-t \Delta_{\mathcal{E}^{\prime}}}\right)=\operatorname{str}_{U_{X}}\left(\varphi\left(\alpha_{X}\right) \mathrm{e}^{-t \Delta_{\mathcal{E}^{\prime}}}\right) .
$$

But since $\alpha_{X}, U_{X}$ and $\left.\Delta_{\mathcal{E}^{\prime}}\right|_{U_{X}}$ are identified with $\alpha, U$ and $\left.\Delta_{\mathcal{E}}\right|_{U}$, respectively, $\varphi(\alpha) \mathrm{e}^{-t \Delta_{\mathcal{E}}}$ makes sense as a trace class operator on $K_{\mathcal{E}_{\left.\right|_{U} L^{2}}}$ for any $t>0$, and

$$
\operatorname{str}_{U}\left(\varphi(\alpha) \mathrm{e}^{-t \Delta_{\mathcal{E}}}\right)=\operatorname{str}_{X}\left(\varphi\left(\alpha_{X}\right) \mathrm{e}^{-t \Delta_{\mathcal{E}^{\prime}}}\right)
$$

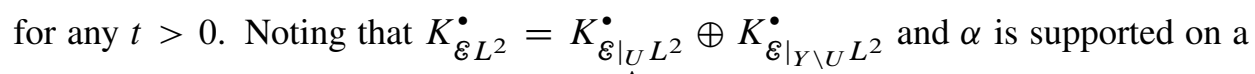
compact subset of $U$, we see that $\varphi(\alpha) \mathrm{e}^{-t \Delta_{\mathscr{E}}}$ makes sense as a trace class operator on $K_{\mathcal{E} L^{2}}^{\bullet}$ for any $t>0$, and

$$
\operatorname{str}_{Y}\left(\varphi(\alpha) \mathrm{e}^{-t \Delta_{\mathcal{E}}}\right)=\operatorname{str}_{X}\left(\varphi\left(\alpha_{X}\right) \mathrm{e}^{-t \Delta_{\mathcal{E}^{\prime}}}\right)
$$

for any $t>0$. This proves part (1).

Step 2: By (4), we have

$$
\lim _{t \rightarrow \infty} \operatorname{str}_{Y}\left(\varphi(\alpha) \mathrm{e}^{-t \Delta_{\mathcal{E}}}\right)=\lim _{t \rightarrow \infty} \operatorname{str}_{X}\left(\varphi\left(\alpha_{X}\right) \mathrm{e}^{-t \Delta_{\mathcal{E}^{\prime}}}\right)
$$

Since $X$ is compact, a trivial modification of the argument proving Proposition 2 will show that the right-hand side is finite. It follows that $\alpha \leadsto \lim _{t \rightarrow \infty} \operatorname{str}\left(\varphi(\alpha) \mathrm{e}^{-t \Delta_{\mathcal{E}}}\right)$ yields a linear functional on the space of compactly supported sections of degree 0 of $\widehat{\operatorname{Hoch}(\mathscr{D i f f}(\mathcal{E}))}$ that are supported on any fixed open disc $U \subset W$ such that $W \subset Y$ is an open disc on which $\mathcal{E}$ is trivial. That this extends to a linear functional on the space of degree 0 elements of $\left.\Gamma_{c}(Y, \widehat{\operatorname{Hoch}(\mathscr{D i f f}(\mathcal{E})})\right)$ follows from a partition of unity argument similar to that used to prove part (1) of this proposition. 
Step 3: To show that $\alpha \leadsto \lim _{t \rightarrow \infty} \operatorname{str}\left(\varphi(\alpha) \mathrm{e}^{-t \Delta_{\mathcal{E}}}\right)$ yields a linear functional on $\left.\mathrm{H}^{0}\left(\Gamma_{c}(Y, \widehat{\operatorname{Hoch}(\mathscr{D i f f}(\mathcal{E})})\right)\right)$, we need to show that $\lim _{t \rightarrow \infty} \operatorname{str}\left(\varphi\left(d_{\mathrm{Hoch}} \beta\right) \mathrm{e}^{-t \Delta_{\mathcal{E}}}\right)=0$ for any degree -1 element $\beta$ of $\left.\Gamma_{c}(Y, \widehat{\operatorname{Hoch}(\mathscr{D i f f}(\mathcal{E})})\right)$. Here, $d_{\text {Hoch }}$ is the differential of the complex $\Gamma_{c}(Y, \widehat{\operatorname{Hoch}(\mathscr{D i f f}(\mathcal{E}))})$. Once more, as in the proof of part (1), one can first show that it suffices to confirm that $\lim _{t \rightarrow \infty} \operatorname{str}\left(\varphi\left(d_{\text {Hoch }} \beta\right) \mathrm{e}^{-t \Delta_{\mathcal{E}}}\right)=0$ for any degree -1 element $\beta$ of $\left.\Gamma_{c}(Y, \widehat{\operatorname{Hoch}(\mathscr{D i f f}(\mathcal{E})})\right)$ supported on a subset of an open disc $U \subset W$ such that $W \subset Y$ is an open disc on which $\mathcal{E}$ is trivial. In this case, if $X$ and $\mathcal{E}^{\prime}$ are as in step 1 of this proof, then

$$
\lim _{t \rightarrow \infty} \operatorname{str}_{Y}\left(\varphi\left(d_{\mathrm{Hoch}} \beta\right) \mathrm{e}^{-t \Delta_{\mathcal{E}}}\right)=\lim _{t \rightarrow \infty} \operatorname{str}_{X}\left(\varphi\left(d_{\mathrm{Hoch}} \beta_{X}\right) \mathrm{e}^{-t \Delta_{\mathcal{E}^{\prime}}}\right) .
$$

Since $X$ is compact, by an easy generalization of Proposition 2, we obtain that

$$
\lim _{t \rightarrow \infty} \operatorname{str}_{X}\left(\varphi\left(d_{\mathrm{Hoch}} \beta_{X}\right) \mathrm{e}^{-t \Delta_{\mathcal{E}^{\prime}}}\right)=\Pi_{K_{0 \mathcal{E}^{\prime}}} \circ \varphi\left(d_{\mathrm{Hoch}} \beta_{X}\right) \circ \mathcal{I}_{K_{0 \mathcal{E}^{\prime}}} \cdot
$$

The right-hand side is precisely $\int_{X}\left[d_{\text {Hoch }} \beta_{X}\right]=0$ by the integral conjecture for compact complex manifolds. This proves part (2) of the proposition.

Note that $\widehat{\operatorname{Hoch}(\mathscr{D i f f}(\mathscr{E}))}$ is a complex of soft sheaves which are modules over the sheaf of smooth functions on $Y$. Furthermore, $\widehat{\operatorname{Hoch}(\mathscr{D i f f}(\mathscr{E}))}$ is quasi-isomorphic to $\left.\overline{C^{\bullet}(\mathcal{D i f f}(\mathscr{E})}\right)$, which in turn is quasi-isomorphic to the shifted constant sheaf $\underline{\mathbb{C}}[2 n]$ (see [Ram], Lemma 3). It follows that $\left.\mathrm{H}^{0}\left(\Gamma_{c}(Y, \widehat{\operatorname{Hoch}(\mathscr{D i f f}(\mathcal{E})})\right)\right) \simeq \mathrm{H}_{c}^{2 n}(Y, \mathbb{C})$. By part (2) of Proposition 6, we have constructed a $\mathbb{C}$-linear functional on $\mathrm{H}_{c}^{2 n}(Y, \mathbb{C})$, which we will denote by $I_{\mathcal{E}}$. The formula for this linear functional on $\mathrm{H}_{c}^{2 n}(Y, \mathbb{C})$ coincides with that for the FLS functional on $\mathcal{E}$ as constructed in [Ram] when $Y$ is compact. The following generalization of Theorem 2 of [Ram] holds.

Theorem 1. Let E be a holomorphic vector bundle having bounded geometry on a connected complex manifold $Y$. Then

$$
I_{\varepsilon}=\int_{Y}: \mathrm{H}_{c}^{2 n}(Y, \mathbb{C}) \rightarrow \mathbb{C} .
$$

Proof. Let $U, W$ be open discs with $U \subset W \subset Y$. Choose a 0-cocycle $\alpha$ of $\Gamma_{c}(Y, \widehat{\operatorname{Hoch}(\mathscr{D i f f}(\mathcal{E}))})$ such that $[\alpha]_{U} \neq 0$ in $\mathrm{H}_{c}^{2 n}(U, \mathbb{C})$. Let $U_{X}, X$ and $\mathcal{E}^{\prime}$ be as in step 1 of the proof of Proposition 6. Then

$$
I_{\mathcal{E}^{\prime}}\left(j_{X_{*}}[\alpha]_{U}\right)=\int_{X} j_{X_{*}}[\alpha]_{U}
$$

by the integral conjecture for compact complex manifolds. But $\int_{X} j_{X_{*}}[\alpha]_{U}=$ 
$\int_{U}[\alpha]_{U}=\int_{Y} j_{Y_{*}}[\alpha]_{U}$. Moreover,

$$
\begin{aligned}
I_{\mathcal{E}}\left(j_{Y_{*}}[\alpha]_{U}\right) & =\lim _{t \rightarrow \infty} \operatorname{str}_{Y}\left(\varphi(\alpha) \mathrm{e}^{-t \Delta_{\mathcal{E}}}\right), \\
I_{\mathcal{E}^{\prime}}\left(j_{X_{*}}[\alpha]_{U}\right) & =\lim _{t \rightarrow \infty} \operatorname{str}_{X}\left(\varphi\left(\alpha_{X}\right) \mathrm{e}^{-t \Delta_{\mathcal{E}^{\prime}}}, .\right.
\end{aligned}
$$

By (4), $I_{\mathcal{E}}\left(j_{Y_{*}}[\alpha]_{U}\right)=I_{\mathcal{E}^{\prime}}\left(j_{X_{*}}[\alpha]_{U}\right)$. It follows that $\int_{Y} j_{Y_{*}}[\alpha]_{U}=I_{\mathcal{E}}\left(j_{Y_{*}}[\alpha]_{U}\right)$. Since $j_{Y_{*}}[\alpha]_{U} \neq 0$ and $\mathrm{H}_{c}^{2 n}(Y, \mathbb{C})$ is a one-dimensional $\mathbb{C}$-vector space, the theorem follows.

Remark. One may define the "completed Hochschild homology with compact sup-

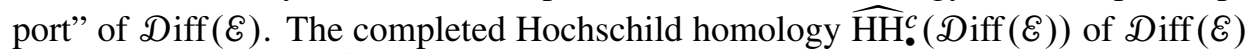
is given by $\left.\mathrm{R} \Gamma_{c}\left(\overline{C^{\bullet}(\mathscr{D i f f}(\mathscr{E})}\right)\right)$. Note that since $\widehat{\operatorname{Hoch}(\mathscr{D i f f}(\mathscr{E}))}$ is a complex of soft sheaves on $X$ quasi-isomorphic to $\left.\overline{C^{\bullet}(\operatorname{Diff}(\mathscr{E})}\right)$, we have

$$
\left.\left.\widehat{\mathrm{HH}_{i}^{c}(\operatorname{Diff}(\mathscr{E})}\right) \simeq \mathrm{H}^{i}\left(\Gamma_{c}(Y, \widehat{\operatorname{Hoch}(\operatorname{Diff}(\mathcal{E})})\right)\right) .
$$

Also note that if $Y$ is compact, then $\Gamma_{c}=\Gamma$. It follows that $\left.\overline{\operatorname{HH}_{i}^{c}(\mathscr{D i f f}(\mathscr{E})}\right)=$ $\left.\widehat{\mathrm{HH}_{i}(\mathscr{D i f f}(\mathcal{E})}\right)$ if $Y$ is compact. It now becomes easy to observe that Proposition 6 extends the definition of $I_{\mathcal{E}}$ to vector bundles on arbitrary manifolds by generalizing the definition of the linear functional tr on $\widehat{\mathrm{HH}}_{0}(\mathscr{D i f f}(\mathcal{E}))$ in the compact case to that of a linear functional on $\overline{\mathrm{HH}_{0}^{c}(\operatorname{Diff}(\mathscr{E}))}$.

Denote by $\left.\overline{\mathrm{CC}^{\bullet}\left(\operatorname{Diff}^{\bullet}(\mathcal{E})(U)\right.}\right)$ the completed Tsygan double complex of $\operatorname{Diff}^{\bullet}(\mathcal{E})(U)$. Denote the sheafification of the pre-sheaf

$$
\left.U \leadsto \operatorname{Tot}\left(\overline{\mathrm{CC}^{\bullet}\left(\operatorname{Diff}^{\bullet}(\mathcal{E})(U)\right.}\right)\right)
$$

by $\widetilde{\operatorname{Cycl}}(\mathscr{D i f f}(\mathcal{E}))$. This is a (soft) sheaf of modules over the sheaf of smooth functions on $Y$ that is quasi-isomorphic to the completed cyclic complex of $\mathscr{D i f f}(\mathcal{E})$. Since the latter complex is quasi-isomorphic to $\underline{\mathbb{C}}[2 n] \oplus \underline{\mathbb{C}}[2 n+2] \oplus \cdots$, we have

$\left.\mathrm{H}^{-2 i}\left(\Gamma_{c}(Y, \widehat{\operatorname{Hoch}(\mathscr{D i f f}(\mathcal{E})})\right)\right) \simeq \mathrm{H}_{c}^{2 n-2 i}(Y, \mathbb{C}) \oplus \mathrm{H}_{c}^{2 n-2 i+2}(Y, \mathbb{C}) \oplus \cdots \oplus \mathrm{H}_{c}^{2 n}(Y, \mathbb{C})$.

One may also note that a $-2 i$ cocycle of $\Gamma_{c}(Y, \widetilde{\operatorname{Cycl}}(\mathscr{D i f f}(\mathscr{E})))$ arises out of a tuple $\left(\beta_{-2 i}, \ldots, \beta_{0}, \ldots, \beta_{l}\right)$, where $\left.\beta_{k} \in \Gamma_{c}(Y, \widehat{\operatorname{Hoch}(\mathscr{D i f f}(\mathcal{E})})^{k}\right)$ if $k$ is even, and $\left.\beta_{k} \in \Gamma_{c}(Y, \widehat{\operatorname{bar}(\mathscr{D i f f}(\mathscr{E})})^{k}\right)$ if $k$ is odd. Note that the terms of the "bar complex" $\widehat{\operatorname{bar}(\mathscr{D i f f}(\mathcal{E})})$ are the same as those of $\widehat{\operatorname{Hoch}(\mathscr{D i f f}(\mathcal{E}))}$, but the differential of the complex $\overline{\operatorname{bar}(\mathscr{D i f f}(\mathcal{E}))}$ is an extension of the bar differential rather than the Hochschild differential. The proof of the following result, which uses Proposition 6, is very similar to that of Proposition 13 of [Ram] and is thus omitted. 
Proposition 7. The map

$$
\left(\beta_{-2 i}, \ldots, \beta_{0}, \ldots, \beta_{l}\right) \leadsto \lim _{t \rightarrow \infty} \operatorname{str}\left(\varphi\left(\beta_{0}\right) \mathrm{e}^{-t \Delta_{\mathcal{E}}}\right)
$$

induces a $\mathbb{C}$-linear functional on $\left.\mathrm{H}^{-2 i}\left(\Gamma_{c}(Y, \widehat{\operatorname{Hoch}(\mathscr{D i f f}(\mathcal{E})})\right)\right)$.

$\mathrm{On}_{c}^{2 n-2 i}(Y, \mathbb{C}) \oplus \mathrm{H}_{c}^{2 n-2 i+2}(Y, \mathbb{C}) \oplus \cdots \oplus \mathrm{H}_{c}^{2 n}(Y, \mathbb{C})$ we have thus constructed a $\mathbb{C}$ - linear functional. We will denote the composition of this $\mathbb{C}$-linear functional with the inclusion of $\mathrm{H}_{c}^{2 n-2 k}(Y, \mathbb{C})$ into $\mathrm{H}_{c}^{2 n-2 i}(Y, \mathbb{C}) \oplus \mathrm{H}_{c}^{2 n-2 i+2}(Y, \mathbb{C}) \oplus \cdots \oplus \mathrm{H}_{c}^{2 n}(Y, \mathbb{C})$ as a direct summand by $I_{\mathcal{E}, 2 i, 2 k}$ whenever $0 \leq k \leq i$. The following generalization of Theorem 3 of [Ram] holds. Its proof completely parallels the proof of Theorem 3 in [Ram], Section 5.

Theorem 2. Let E be a holomorphic vector bundle having bounded geometry on a complex manifold $Y$. Then

$$
I_{\mathcal{E}, 2 i, 0}=\int_{Y}: \mathrm{H}_{c}^{2 n}(Y, \mathbb{C}) \rightarrow \mathbb{C}
$$

for any $i \geq 0$. Furthermore,

$$
I_{\mathcal{E}, 2 i, 2 k}=0 \text { for all } 0<k \leq i .
$$

Remark. One may define the "completed cyclic homology with compact support" $\widehat{\mathrm{HC}_{\bullet}^{c}(\mathscr{D i f f}(\mathcal{E}))}$. By definition, $\left.\left.\overline{\operatorname{HC}_{\bullet}^{c}(\mathscr{D i f f}(\mathcal{E})}\right)=\mathrm{R} \Gamma_{c}(Y, \overline{\operatorname{Cycl}(\mathscr{D i f f}(\mathscr{E})})\right)$ where $\widehat{\operatorname{Cycl}(\mathscr{D i f f}(\mathscr{E})})$ is the completed cyclic chain complex of $\mathscr{D i f f}(\mathcal{E})$. As $\widetilde{\operatorname{Cycl}}(\mathscr{D i f f}(\mathcal{E}))$ is quasi-isomorphic to $\overline{\operatorname{Cycl}(\mathscr{D i f f}(\mathcal{E}))}$ and is a complex of soft sheaves on $Y$, it follows that

$$
\left.\widehat{\operatorname{HC}_{i}^{c}(\mathscr{D i f f}(\mathcal{E})}\right) \simeq \mathrm{H}^{i}\left(\Gamma_{c}(Y, \widetilde{\operatorname{Cycl}}(\mathscr{D i f f}(\mathcal{E})))\right) .
$$

Then $I_{\mathcal{E}, 2 i, 2 k}$ is constructed in the non-compact case by observing that the construction of $\operatorname{tr}_{2 i}$ in the compact case generalizes to yield a linear functional on $\left.\overline{\mathrm{HC}_{-2 i}^{c}(\mathcal{D i f f}(\mathscr{E})}\right)$ for each $i \geq 0$ by Proposition 7 .

\section{References}

[BGV] N. Berline, E. Getzler, and M. Vergne, Heat kernels and Dirac operators. Grundlehren Text Editions, Springer-Verlag, Berlin 2004. Zbl 1037.58015 MR 2273508

[Bryl] J.-L. Brylinski, A differential complex for Poisson manifolds. J. Differential Geom. 28 (1988), 93-114. Zbl 0634.58029 MR 950556

[BrGe] J.-L. Brylinski and E. Getzler, The homology of algebras of pseudo-differential symbols and the noncommutative residue. K-Theory 1 (1987), 385-403. Zbl 0646.58026 MR 0920951 
[Don] Donelly, H., Asymptotic expansions for the compact quotients of properly discontinuous group actions. Illinois J. Math. 23 (1979), 485-496. Zbl 0411.53033 MR 0537804

[EnFe] M. Engeli and G. Felder, A Riemann-Roch-Hirzebruch formula for traces of differential operators. Preprint 2007. arXiv:math.QA/0702461

[FLS] B. Feigin, A. Losev, and B. Shoikhet, Riemann-Roch-Hirzebruch theorem and topological quantum mechanics. Preprint 2004. arXiv:math.QA/0401400

[FT] B. L. Feigin and B. L. Tsygan, Riemann-Roch theorem and Lie algebra cohomology, I. Rend. Circ. Mat. Palermo (2) Suppl. 21 (1989), 15-52. Zbl 0686.14007 MR 1009564

[Lod] J.-L. Loday, Cyclic homology. 2nd ed., Grundlehren Math. Wiss. 301, Springer-Verlag, Berlin 1998. Zbl 0885.18007 MR 1600246

[NT1] R. Nest and B. Tsygan, Algebraic index theorem. Comm. Math. Phys. 172 (1995), 223-262. Zbl 0887.58050 MR 1350407

[NT2] R. Nest and B. Tsygan, On the cohomology ring of an algebra. In Advances in geometry, Progr. Math. 172, Birkhäuser, Boston 1999, 337-370. Zbl 0919.57019 MR 1667686

[Ram] A. C. Ramadoss, Some notes on the Feigin-Losev-Shoikhet integral conjecture. $J$. Noncommut. Geom. 2 (2008), 405-448.

[Riet] K. Rietsch, An introduction to perverse sheaves. In Representations of finite dimensional algebras and related topics in Lie theory and geometry, Fields Inst. Commun. 40, Amer. Math. Soc., Providence, RI, 2004, 391-429. Zbl 1058.55002 MR 2057405

[S-S] P. Schapira and J.-P. Schneiders, Index theorem for elliptic pairs. Astérisque 224 (1994). Zbl 0856.58037 MR 1305641

[Vois] C. Voisin, Hodge theory and complex algebraic geometry I. Cambridge Stud. Adv. Math. 76, Cambridge University Press, Cambridge 2002. Zbl 1005.14002 MR 1967689

Received December 9, 2007; revised March 11, 2008

A. C. Ramadoss, Department of Mathematics, University of Oklahoma, Norman, OK 73019-0315, U.S.A.

E-mail: aramadoss@math.ou.edu 